\title{
ON BOUNDEDNESS OF COMPOSITION OPERATORS ON $H^{2}\left(B_{2}\right)$
}

\author{
JOSEPH A. CIMA, CHARLES S. STANTON AND WARREN R. WOGEN
}

\begin{abstract}
Composition operators on the Hardy space $H^{2}$ of the ball in $\mathrm{C}^{2}$ are studied. Some sufficient conditions are given for a composition operator to be bounded. A class of inner mappings is given which induces isometric composition operators. Another class of inner mappings is shown to induce unbounded composition operators.
\end{abstract}

1. Introduction and definitions. Let $B_{n}$ be the unit ball in $\mathrm{C}^{n}$ and let $H^{p}=H^{p}\left(B_{n}\right)$ be the Hardy space on $B_{n}$. For $p \geqslant 1, H^{p}$ is a Banach space. If $\phi: B_{n} \rightarrow B_{n}$ is holomorphic, then $\phi$ induces the linear operator $C_{\phi}$ from $H^{p}$ into the holomorphic functions on $B_{n}$ given by $C_{\phi} f=f \circ \phi . C_{\phi}$ is the composition operator induced by $\phi$.

Definitive results on composition operators are known for the case $n=1$ (see [3] and the references given therein). In particular, for $n=1, C_{\phi}$ is always a bounded operator on $H^{p}$. One can check the integral condition on $f \circ \phi$ or observe that the class of positive harmonic functions is invariant under holomorphic change of variables. In addition, subordination techniques can be used to compare the integral means of $f$ and $f \circ \phi$.

The proofs that every $C_{\phi}$ is bounded for $n=1$ fail if $n \geqslant 2$. In fact, J. H. Shapiro has given examples of holomorphic mappings $\phi$ of $B_{n}$ into $B_{n}(n \geqslant 2)$ for which $C_{\phi}$ is not bounded on $H^{2}$. Note that although we still have the existence of certain positive majorants (harmonic, $M$-harmonic), these classes of majorants are not invariant under holomorphic change of variables.

The results of this paper are of two types. We show that a certain class of inner mappings induces unbounded composition operators on $H^{2}$. We also give some sufficient conditions that $C_{\phi}$ be bounded. We give examples in $\$ 4$ which answer in the affirmative the following question of Rudin [5]: “Is there an inner mapping $\phi$ of $B_{n}$ into $B_{n}$, not an automorphism of $B_{n}$, which is measure-preserving?" If $\phi$ is measure-preserving, then $C_{\phi}$ is an isometry from $H^{p}$ into $H^{p}$. We remark that for $1 \leqslant p \leqslant \infty, p \neq 2$, the onto isometries of $H^{p}$ are known (cf. [4, p. 152]).

Finally, we note that B. MacCluer [1] has studied $C_{\phi}$ when $\phi$ is a holomorphic automorphism of $B_{n}$. She has also studied the spectra of compact composition operators and has given some examples of unbounded composition operators in [2].

Received by the editors May 23, 1983 and, in revised form, July 13, 1983. The first author presented a talk on the contents of this paper at the Summer Meeting of the American Mathematical Society, August 8-12, 1983 in Albany, New York.

1980 Mathematics Subject Classification. Primary 47B99, 32A35. 
We restrict our attention to $p=2$ and, for notational convenience, to $n=2$. Let $B=\left\{z=\left(z_{1}, z_{2}\right):\left|z_{1}\right|^{2}+\left|z_{2}\right|^{2}<1\right\}$ and let $S$ be the boundary of $B$. Let $d \sigma$ denote normalized surface measure on $S$ and let $d v$ denote volume measure on $B$. A holomorphic function $f$ on $B$ is in $H^{2}$ if

$$
\sup _{0<r<1} \int_{S}|f(r \zeta)|^{2} d \sigma(\zeta) \equiv\|f\|^{2}<\infty
$$

It is known [4, p. 84] that $f$ is in $H^{2}$ if and only if $|f|^{2}$ has a harmonic majorant if and only if $|f|^{2}$ has an $M$-harmonic majorant. (Recall that $u: B \rightarrow \mathrm{C}$ is $M$-harmonic if $\tilde{\Delta} u=0$, where $\tilde{\Delta}$ is the invariant Laplacian [4, p. 47].) In the latter case, there is a least $M$-harmonic majorant of the form

$$
u(z)=\int_{S} P(z, \zeta) h(\zeta) d \sigma(\zeta), \quad z \in B, h \in L^{2}(S)
$$

and

$$
P(z, \zeta)=\left(\left(1-|z|^{2}\right) /|1-\langle z, \zeta\rangle|^{2}\right)^{2}
$$

All mappings are assumed to be holomorphic. A mapping $\phi: B \rightarrow B$ is inner if for almost every $\zeta \in S$, the radial $\operatorname{limit}_{\lim _{r \rightarrow 1}} \phi(r \zeta)=\phi(\zeta)$ is in $S$.

Finally, we recall [3] that if $\phi$ is a mapping of $B_{1}$ to $B_{1}$, then

$$
\left\|C_{\phi}\right\|^{2} \leqslant(1+|\phi(0)|) /(1-|\phi(0)|)
$$

2. Unbounded composition operators. The first example known to us of a mapping $\phi$ for which $C_{\phi}$ is unbounded was given by J. H. Shapiro (private communication). Let $\phi\left(z_{1}, z_{2}\right)=\left(2 z_{1} z_{2}, 0\right)$. Then $\phi$ maps $B$ onto a slice, and $\phi$ has vanishing Jacobian. Further, for $\zeta \in S$, we have $|\phi(\zeta)|=1$ precisely when $\zeta$ is on the torus $\left\{2^{-1 / 2}\left(e^{i \theta_{1}}, e^{i \theta_{2}}\right): \theta_{1}, \theta_{2} \in[0,2 \pi)\right\}$. We sketch a proof that $C_{\phi}$ is unbounded. The set $\left\{f_{j, k}\left(z_{1}, z_{2}\right)=z_{1}^{j} z_{2}^{k}: j, k \geqslant 0\right\}$ is an orthogonal basis for $H^{2}$, and

$$
\left\|f_{j, k}\right\|^{2}=j ! k ! /(1+j+k) ! .
$$

Now $C_{\phi} f_{j, 0}=2^{j} f_{j, j}$, and an application of Stirling's formula will show that

$$
\lim _{j \rightarrow \infty} \frac{\left\|C_{\phi} f_{j, 0}\right\|}{\left\|f_{j, 0}\right\|}=\infty .
$$

(See [2] for a more general version of this example.)

We present another simple example of an unbounded composition operator. Let $\psi$ be an inner function and let $\phi\left(z_{1}, z_{2}\right)=\left(\psi\left(z_{1}, z_{2}\right), 0\right)$. Then $C_{\phi}$ is unbounded. (This example also appears in [2] with a different proof of unboundedness.)

Since automorphisms of $B$ induce bounded composition operators, we may assume $\phi(0)=0$. Choose $f \in H^{2}$ for which $\int_{0}^{2 \pi}\left|f\left(e^{i \theta}, 0\right)\right|^{2} \mathrm{~d} \theta=\infty$. For $\zeta \in S$, let $\phi_{\zeta}\left(e^{i \theta}\right)=\phi\left(e^{i \theta} \zeta\right)$. Then for almost every $\zeta, \phi_{\zeta}$ is inner and $\phi_{\zeta}(0)=0$. Thus (see [3]) 
$\phi_{\zeta}$ induces an isometry on $H^{2}\left(B_{1}\right)$. Hence (see [4, Proposition 1.4.7(1)]),

$$
\begin{aligned}
\int_{S}|f \circ \phi|^{2} d \sigma & =\int_{S} d \sigma(\zeta) \frac{1}{2 \pi} \int_{0}^{2 \pi}\left|f\left(\phi_{\zeta}\left(e^{i \theta}\right)\right)\right|^{2} d \theta \\
& =\frac{1}{2 \pi} \int_{0}^{2 \pi}\left|f\left(e^{i \theta}, 0\right)\right|^{2} d \theta=\infty
\end{aligned}
$$

We generalize this example as follows.

TheOREM 1. Suppose $(A, B) \in S$ and $\phi_{1}$ and $\phi_{2}$ are inner functions on $B$. If $\phi\left(z_{1}, z_{2}\right)=\left(A \phi_{1}\left(z_{1}, z_{2}\right), B \phi_{2}\left(z_{1}, z_{2}\right)\right)$, then $C_{\phi}$ is unbounded.

Proof. The theorem is true for $A=0$ or $|A|=1$, so let $r=|A|^{2}$ and suppose $0<r<1$. For every positive integer $N$, there is an integer $K_{N}$ so that $\left(K_{N}-1\right) / N$ $\leqslant r<K_{N} / N$. Thus $\left(K_{N}-1\right) / K_{N} \leqslant N r / K_{N}<1$, so

$$
\left(1-1 / K_{N}\right)^{K_{N}}<\left(N r / K_{N}\right)^{K_{N}}<1 \text {. }
$$

Choose $N_{0}$ so that if $N \geqslant N_{0}$, then $K_{N} \geqslant 2$. For $N \geqslant N_{0}$, we have $\frac{1}{4}<$ $\left(1-1 / K_{N}\right)^{K_{N}}$. Thus by $(2), r^{K_{N}}>\frac{1}{4}\left(K_{N} / N\right)^{K_{N}}$. Also note that $1-K_{N} / N<1-r$. Then

$$
\left\|f_{K_{N}, N-K_{N}} \circ \phi\right\|^{2}=r^{K_{N}}(1-r)^{N-K_{N}} \geqslant \frac{1}{4}\left(\frac{K_{N}}{N}\right)^{K_{N}}\left(\frac{N-K_{N}}{N}\right)^{N-K_{N}} .
$$

We use the following form of Stirling's formula:

$$
n !=\left(n^{n} / e^{n}\right) \sqrt{n} e^{c_{n}}, \quad \text { with } e \geqslant e^{c_{n}} \geqslant e / \sqrt{2} .
$$

Now by (3) and Stirling's formula,

$$
\begin{aligned}
\frac{\left\|f_{K_{N}, N-K_{N}} \circ \phi\right\|^{2}}{\left\|f_{K_{N}, N-K_{N}}\right\|^{2}} \geqslant & C_{N}\left(\frac{1}{4}\right)\left(\frac{K_{N}}{N}\right)^{K_{N}}\left(\frac{N-K_{N}}{N}\right)^{N-K_{N}} \\
& \times \frac{(N+1)^{N+1} \sqrt{N+1} e^{K_{N}} e^{N-K_{N}}}{e^{N+1}\left(K_{N}\right)^{K_{N}} \sqrt{K_{N}}\left(N-K_{N}\right)^{N-K_{N}} \sqrt{N-K_{N}}} \\
\geqslant & \frac{C_{N}}{4}\left(\frac{N+1}{N}\right)^{N} \frac{(N+1)^{3 / 2}}{\sqrt{K_{N}\left(N-K_{N}\right)}}>\frac{C_{N}}{4} \sqrt{N} .
\end{aligned}
$$

Hence $C_{\phi}$ is unbounded.

We remark that we can choose the inner mapping $\phi$ of Theorem 1 so that its Jacobian is not identically zero. However, the range of $\phi$ is contained in a polydisc, so that $\phi$ is not onto $B$.

3. Bounded composition operators. The linear mapping $\phi\left(z_{1}, z_{2}\right)=\left(z_{1}, 0\right)$ has the same range as Shapiro's example, has vanishing Jacobian, and induces a bounded composition operator. This can be deduced by [4, p. 127] or from the following proposition. (See [5] for the polydisc version.) 
Proposition 1. If $\phi$ is a mapping from $B$ into $B$ and if $\phi\left(z_{1}, z_{2}\right)=\left(\phi_{1}\left(z_{1}\right), \phi_{2}\left(z_{2}\right)\right)$, then $C_{\phi}$ is bounded.

Proof. As before, we may assume $\phi(0)=0$. Fix $r<1$ and let $f \in H^{2}$. Then (see $[4$, p. 15])

$$
\int_{S}|f \circ \phi(r \zeta)|^{2} d \sigma(\zeta)=\int_{S}\left|\frac{1}{2 \pi} \int_{0}^{2 \pi} f\left(\phi_{1}\left(r e^{i \theta} \zeta_{1}\right), \phi_{2}\left(r \zeta_{2}\right)\right) d \theta\right|^{2} d \sigma(\zeta)
$$

Fix $\zeta_{2}$ and apply (1). Then

$$
\int_{S}|f \circ \phi(r \zeta)|^{2} d \sigma(\zeta) \leqslant \int_{S} \frac{1}{2 \pi} \int_{0}^{2 \pi}\left|f\left(r e^{i \theta} \zeta_{1}, \phi_{2}\left(r \zeta_{2}\right)\right)\right|^{2} d \theta d \sigma(\zeta) .
$$

Repeating this argument in the second coordinate gives $\|f \circ \phi\|^{2} \leqslant\|f\|^{2}$.

A mapping on $B$ of the form $\phi\left(z_{1}, z_{2}\right)=\left(z_{1}^{j} / a, z_{2}^{k} / b\right)$ has a "generalized ellipsoid" as its range. If $\phi$ maps into $B$, then Proposition 1 shows $C_{\phi}$ is bounded.

The following theorem gives another sufficient condition for boundedness of $C_{\phi}$. We use the notation $J \phi=\left(D_{i} \phi_{j}\right)_{i, j=1}^{2}$ for the complex Jacobian matrix of the mapping $\phi$, and $|J \phi|$ is the absolute value of the determinant of $J \phi$. Also $\Delta$ denotes the Laplacian.

THEOREM 2. Let $\phi$ be a one-to-one mapping of $B$ into $B$. Suppose there are positive constants $c$ and $M$ so $|J \phi(z)| \geqslant c$ on $B$ and $\left|D_{i} \phi_{j}(z)\right| \leqslant M$ on $B$, for $i, j=1,2$. Then $C_{\phi}$ is bounded.

Proof. As before, we assume $\phi(0)=0$. Assume $f$ is holomorphic on $B$ and $0<r<1$. Let $g_{r}(z)=|z|^{-2}-r^{-2}$ and let $B(r)=\left\{z \in \mathbf{C}^{2}:|z|<r\right\}$. Apply Green's Theorem to conclude that

$4 \pi^{2} \int_{S}\left(g_{r}(r \zeta) \frac{\partial|f(r \zeta)|^{2}}{\partial r}-|f(r \zeta)|^{2} \frac{\partial g_{r}(r \zeta)}{\partial r} r^{3}\right) d \sigma(\zeta)=\int_{B(r)}\left(g_{r} \Delta|f|^{2}-|f|^{2} \Delta g_{r}\right) d v$

Then

$$
8 \pi^{2} \int_{S}|f(r \zeta)|^{2} d \sigma(\zeta)=\int_{B(r)} g_{r} \Delta|f|^{2} d v+8 \pi^{2}|f(0)|^{2}
$$

By integration by parts we obtain

$$
\int_{S}|f(r \zeta)|^{2} d \sigma(\zeta)=\frac{1}{8 \pi^{2}} \int_{0}^{r} \rho^{-3}\left[\int_{B(\rho)} \Delta|f|^{2} d v\right] d \rho+|f(0)|^{2} .
$$

Now $\phi(B(r)) \subset B(r)$ by the Schwarz Lemma, and $\Delta|f|^{2} \geqslant 0$, so

$$
\begin{aligned}
\int_{B(r)} \Delta|f|^{2} d v & \geqslant \int_{\phi(B(r))} \Delta|f|^{2} d v=\int_{B(r)}\left[\Delta|f|^{2} \circ \phi\right]|J \phi|^{2} d v \\
& =\sum_{j=1}^{2} \int_{B(r)}\left|D_{j} f\right|^{2}(\phi)|J \phi|^{2} d v
\end{aligned}
$$


Also

$$
\int_{B(r)} \Delta|f \circ \phi|^{2} d v=\int_{B(r)} \sum_{j=1}^{2}\left|\sum_{i=1}^{2} D_{i} f(\phi) D_{j} \phi_{i}\right|^{2} d v
$$

Now we use our hypothesis to conclude that

$$
\frac{C^{2}}{4 M^{2}} \int_{B(r)} \Delta|f \circ \phi|^{2} d v \leqslant \int_{B(r)} \Delta|f|^{2} d v .
$$

Hence from (3), there is a constant $A$ so that $\|f \circ \phi\|^{2} \leqslant A\|f\|^{2}$.

Note that equation (3) in the proof of Theorem 2 is Jensen's formula for subharmonic functions applied to $|f|^{2}$.

ExAmPLE. Let $g$ be a holomorphic function from $B$ into the unit disc and define $\phi\left(z_{1}, z_{2}\right)=g\left(z_{1}, z_{2}\right)\left(z_{1}, z_{2}\right) . \phi$ maps $B$ into $B$. If $\zeta \in S$, then $\phi$ maps the slice $\{\lambda \zeta$ : $|\lambda| \leqslant 1\}$ into itself. If $f \in H^{2}$ and $f_{\zeta}(\lambda)=f(\lambda \zeta)$, then

$$
\|f\|^{2}=\int_{S}|f(\zeta)|^{2} d \sigma(\zeta)=\int_{S} \frac{1}{2 \pi} \int_{0}^{2 \pi}\left|f\left(e^{i \theta} \zeta\right)\right|^{2} d \theta d \sigma(\zeta)=\int_{S}\left\|f_{\zeta}\right\|^{2} d \sigma(\zeta)
$$

For fixed $\zeta \in S$, we have $f(\phi(\lambda \zeta))=f_{\zeta}\left(\lambda g_{\zeta}(\lambda)\right)$. So by (1),

$$
\frac{1}{2 \pi} \int_{0}^{2 \pi}\left|f_{\zeta}\left(e^{i \theta} g_{\zeta}\left(e^{i \theta}\right)\right)\right|^{2} d \theta \leqslant\left\|f_{\zeta}\right\|^{2}
$$

Thus $\|f \circ \phi\|^{2} \leqslant\|f\|^{2}$, and $\left\|C_{\phi}\right\|=1$.

If the function $g$ of the Example is an inner function, then $\phi$ is an inner mapping which is not an automorphism but such that $C_{\phi}$ is bounded. In fact, we will see from Theorem 3 that $C_{\phi}$ is an isometry.

4. Measure-preserving inner mappings. An inner mapping $\phi$ is called measure-preserving if for all Borel subsets $E$ of $S$, we have $\sigma(E)=\sigma\left(\phi^{-1}(E)\right)$.

PROPOSITION 2. $\phi$ is measure-preserving if and only if

$$
\int_{S} f_{j, k}(z) \bar{f}_{m, n}(z) d \sigma(z)=\int_{S} f_{j, k}(\phi(z)) \bar{f}_{m, n}(\phi(z)) d \sigma(z),
$$

for all $j, k, m, n \geqslant 0$.

The proof is straightforward. Use change of variables, the Riesz Representation Theorem for $C(S)$, and the Stone-Weierstrass Theorem.

Since the $f_{j, k}$ 's form an orthogonal basis for $H^{2}$, we have

COROLlaRY 1. $\phi$ is measure-preserving if and only if $C_{\phi}$ is an isometry.

LEMMA 1. If $\psi \in H^{\infty}$ and either $j>m$ or $k>n$, then $\left\langle f_{j, k} \psi, f_{m, n}\right\rangle=0 .(\langle\cdot, \cdot\rangle$ denotes the inner product in $H^{2}$.) 
Proof. Let $\nu$ denote area measure in C. Suppose $j>m$. Then

$$
\begin{aligned}
\left\langle f_{j, k} \psi, f_{m, n}\right\rangle= & \int_{\left|z_{2}\right|<1} z_{2}^{k} \bar{z}_{2}^{n}\left(1-\left|z_{2}\right|^{2}\right)^{(j+m) / 2} \\
& \times\left[\frac{1}{2 \pi} \int_{0}^{2 \pi} \psi\left(\sqrt{1-\left|z_{2}\right|^{2}} e^{i \theta}, z_{2}\right) e^{i(j-m) \theta} d \theta\right] d \nu\left(z_{2}\right)=0,
\end{aligned}
$$

since the inside integral vanishes. The case $k>n$ is handled similarly.

Theorem 3. Suppose $\psi$ is an inner function on $B$ and $M$ and $N$ are nonnegative integers. If $\phi\left(z_{1}, z_{2}\right)=\left(z_{1} \psi^{M}\left(z_{1}, z_{2}\right), z_{2} \psi^{N}\left(z_{1}, z_{2}\right)\right)$, then $\phi$ is a measure-preserving inner mapping.

Proof. First note that $f_{j, k} \circ \phi=f_{j, k} \psi^{M j+N k}$. If $(j, k)=(m, n)$, then (4) clearly holds. If $(j, k) \neq(m, n)$, then

$$
\left\langle f_{j, k} \circ \phi, f_{m, n} \circ \phi\right\rangle=\left\langle f_{j, k} \psi^{M(j-m)+N(k-n)}, f_{m, n}\right\rangle .
$$

If the exponent on $\psi$ is $>0$, then Lemma 2 shows that this inner product is $0=\left\langle f_{j, k}, f_{m, n}\right\rangle$. Thus (4) holds. If the exponent is $<0$, write the inner product as $\left\langle f_{j, k}, f_{m, n} \psi^{M(m-j)+N(n-k)}\right\rangle$ and apply Lemma 1 .

We remark that the class of measure-preserving inner mappings is a semigroup under composition. Thus we can use Theorem 3 to generate other examples of measure-preserving maps (and isometric composition operators).

Finally, we note that all of the questions considered in this paper have analogues for the polydisc. One can show, for instance, that not every holomorphic self-map of the polydisc induces a bounded composition operator. In fact (see [6]) if $\phi\left(z_{1}, z_{2}\right)=$ $\left(z_{1}, z_{1}\right)$, then $C_{\phi}$ is not bounded on $H^{2}$ of the bidisc.

ADDED IN PROOF. B. Tomaszewski has shown in a preprint entitled Interpolation and inner maps that preserve measure, that for $n \geqslant m \geqslant 1$ there exist inner mappings $\Phi ; B^{n} \rightarrow B^{m}$ which are measure-preserving.

\section{REFERENCES}

1. B. MacCluer, Iterates of holomorphic self-maps of the unit ball in $\mathbf{C}^{N}$, Michigan Math. J. 30 (1983), $97-106$.

2. S__ Spectra of compact composition operators on $H^{P}\left(B_{N}\right)$, preprint.

3. E. Nordgren, Composition operators on Hilbert spaces, Hilbert Space Operators, Lecture Notes in Math., Vol. 693, Springer-Verlag, Berlin, 1978, pp. 37-63.

4. W. Rudin, Function theory in the unit ball of $\mathbf{C}^{N}$, Grundelehren Math. Wiss., Vol. 241, Springer-Verlag, Berlin and New York, 1980.

5. Inner functions in the unit ball of $\mathbf{C}^{M}$, J. Funct. Anal. 50 (1983), 100-126.

6. S. D. Sharma and R. K. Singh, Composition operators and several complex variables, Bull. Austral. Math. Soc. 23 (1981), 237-247.

Department of Mathematics, University of North Carolina, Chapel Hill, North Carolina 27514 